\title{
The impact of inter-fraction changes for perineal template-based interstitial gynecologic brachytherapy implants
}

\author{
Jean-Guy Belliveau, PhD!, Kundan Thind, PhD ${ }^{1,2,3}$, Robyn Banerjee, MD ${ }^{3,4}$, Sarah Quirk, PhD',2,3, Corinne Doll, MD 3.4 , \\ Tien Phan, MD3,4, Tyler Meyer, PhD',2,3, Michael Roumeliotis, PhD',2,3 \\ 'Division of Medical Physics, Tom Baker Cancer Centre, Calgary, Canada, ²Department of Physics \& Astronomy, University of Calgary, Calgary, \\ Canada, ${ }^{3}$ Department of Oncology, University of Calgary, Calgary, Canada, ${ }^{4}$ Division of Radiation Oncology, Tom Baker Cancer Centre, \\ Calgary, Canada
}

\begin{abstract}
Purpose: Perineal template-based interstitial gynecologic brachytherapy (ISBT) treatments are evaluated to determine whether adaptive inter-fraction re-planning is beneficial and necessary to meet the treatment aims of the American Brachytherapy Society (ABS) consensus guidelines for interstitial brachytherapy. Adherence to the EMBRACE II protocol is also assessed.

Material and methods: Ten patients receiving radical intent treatment for locally advanced or recurrent gynecologic malignancies underwent a three-fraction ISBT treatment with an ABS-recommended prescription regimen of 21 to 24 Gy. Clinical treatment plans were created according to a computed tomography (CT) acquired immediately post-implant. The first fraction was delivered on the same day as the implant (Day 1). The remaining two fractions were delivered on the next day (Day 2), at least six hours apart. Prior to treating on Day 2, a verification CT was acquired, permitting assessment of over-night changes. The Day 2 CT was used to evaluate deviations in 2-Gy-per-fraction equivalent dose $\left(\mathrm{EQD}_{2}\right)$ from the clinically intended dosimetry for clinical target volume (CTV), bladder, rectum, and sigmoid.

Results: For all patients, the median (range) difference between the intended and the delivered dosimetry for the CTV D90\% was $1.4 \mathrm{~Gy}_{10}\left(0.3-4.4 \mathrm{~Gy}_{10}\right)$. For all normal tissues, the median (range) difference from the intended normal tissue dose was $2.6 \mathrm{~Gy}_{3}\left(0.1-15.5 \mathrm{~Gy}_{3}\right)$. In all cases, the deviation from clinically intended dosimetry did not lead to a violation of recommended normal tissue dose guidelines. For two of 10 patients with large normal tissue differences ( $>10 \mathrm{~Gy}_{3}$ from the intended dose), inter-fraction adaptive planning did improve the plan quality, but was not strictly required to meet the normal tissue dose planning aims.

Conclusions: The implementation of perineal template-based ISBT treatment without inter-fraction adaptive planning can be delivered to comply with the ABS normal tissue dose guidelines and EMBRACE II limits for prescribed dose.

J Contemp Brachytherapy 2019; 11, 2: 122-127 DOI: https://doi.org/10.5114/jcb.2019.84420
\end{abstract}

Key words: brachytherapy, interstitial, adaptive, gynecologic, EMBRACE, inter-fraction, template.

\section{Purpose}

For patients with advanced or recurrent gynecologic malignancies, interstitial brachytherapy (ISBT) has been shown to improve local control, while maintaining an acceptable toxicity profile [1]. Patients with complex and bulky disease are often indicated for the brachytherapy component to be delivered using a perineal template to guide the ISBT implant. Though treatment regimens vary, the application of the prescription dose is commonly delivered over three to six fractions from a single perineal implant that is sutured to the patient $[1,2,3]$. These fractions are delivered a minimum of six hours apart, with the total treatment time spanning multiple, consecutive days. To aid in the safe adoption of complex high-doserate (HDR) brachytherapy gynecologic treatments, consensus-based commissioning guidelines provide a standardized approach to minimize treatment errors [4]; however, the availability of institutional resources create differences in practical clinical implementation, which often impacts the possibility of creating treatment plans on a per fraction basis. Institutional resources are tied to the availability of radiation oncologists, dosimetrists, radiation therapists, and medical physicists, collectively working to treat the patient. Infrastructure constraints including operating room time, imaging resources, nursing, and
Address for correspondence: Michael Roumeliotis, PhD, Division of Medical Physics, Tom Baker Cancer Centre, Calgary AB, T2N 4N2, 133129 Street NW, Calgary, AB, Canada, phone: +1 (403) 521-3789, e-mail: mb.roumeliotis@gmail.com
Received: 24.09 .2018

Accepted: 18.02 .2019

Published: 29.04.2019 
anesthesia support also factor into the complexity of this workflow.

For perineal template-based ISBT, it is a common practice to create a treatment plan for the patient based on imaging simulation immediately preceding the first fraction. This treatment plan is then applied to all subsequent fractions. Previous gynecologic brachytherapy studies have investigated the dosimetric impact of inter-fraction changes [5,6] and the added value of adaptive planning $[7,8,9,10]$. Most of these studies have assessed the value of adaptive planning for treatment regimens where the prescription dose is delivered over five or six fractions $[7,8,9]$. These studies showed mixed results on the benefit of adaptive planning but demonstrate improvement in selected cases with large anatomical changes. For lower fraction treatment regimens, it is intuitive that the inter-fraction changes may have a more considerable cumulative impact on patient dosimetry due to fewer averaging effects. In 2018, Gladwish et al. investigated the application of adaptive planning to a three-fraction treatment regimen, in which the original treatment plan was applied to both fraction one and two, with an additional computed tomography (CT) dataset acquired before the third fraction, which was used to create a new treatment plan for the remaining fraction [10]. The study indicated that, generally, the clinical target volume (CTV) D90\% and $\mathrm{V} 100 \%$ coverage was improved when the adaptive planning strategy was applied. At our institution, a similar three-fraction treatment regimen spanning two days is prescribed; however, the second CT dataset is acquired before the treatment of the second fraction.

In this work, a clinical implementation for perineal template-based ISBT delivered is evaluated to determine whether adaptive re-planning is beneficial and necessary to meet the treatment aims of the American Brachytherapy Society (ABS) consensus guidelines for interstitial brachytherapy [1]. The EMBRACE II clinical trial [11] dosimetry plan has a well-established link to local control, overall survival, and normal tissue toxicity [12]. Although the EMBRACE II was designed to guide planning for intracavitary cervix patients, the protocol can also be used to guide normal tissue constraints for primary vaginal cancers or endometrial cancers with a vaginal recurrence. These normal tissue constraints are more strict than the ABS consensus guidelines [1]. The presented approach provides practitioners with a clinical framework for the implementation of perineal template-based IBST treatment planning that can be applied to safely meet the ABS and the EMBRACE II normal tissue constraints to achieve acceptably low normal tissue toxicity without the routine use of adaptive planning.

\section{Material and methods}

\section{Patients' population}

From September 2016 to January 2018, 10 patients receiving radical intent treatment for locally advanced or recurrent gynecologic malignancies were treated according to a three-fraction perineal template-based ISBT prescription regimen. All patients were treated with a prescription regimen of 21 to $24 \mathrm{~Gy}$, which is consistent with the American Brachytherapy Society consensus guidelines $[1,13]$. The variability in prescribed dose was based on physician expectation for tolerance to normal tissue doses. Prior to brachytherapy, all patients underwent external beam radiotherapy to the pelvis of $45 \mathrm{~Gy}$ in 25 fractions. Characteristics of the patients' population are shown in Table 1

\section{Clinical implementation of three-fraction perineal template image-guided brachytherapy}

For the brachytherapy component of treatment, each patient was required to have a perineal template implant in lithotomy position that was guided by trans-rectal (Endocavity Biplane 8848) ultrasound imaging (BK Medical, Copenhagen, Denmark) to place 15 gauge Flexineedles via a Syed-Neblett template (Best ${ }^{\mathrm{TM}}$ Medical International, Springfield, VA, USA). Ultrasound images were generally acquired at $9 \mathrm{MHz}$, with imaging depth selected to accommodate patient anatomy. After implantation, patient leg positioning was relaxed from the lithotomy position. For all patients, the first fraction was delivered on the same day as the implant (Day 1). The remaining two fractions were delivered on the next day (Day 2), at least six hours apart. Prior to each CT image acquisition or fraction delivery, each patient had their bladder filled to $180 \mathrm{~cm}^{3}$ via Foley catheter. Bladder consistency was verified by CT imaging, with all images acquired on the Philips Big Bore (Philips, Amsterdam, The Netherlands) at $120 \mathrm{kVp}, 200 \mathrm{mAs}$, and $3 \mathrm{~mm}$ slice thickness.

Figure 1A depicts the following treatment workflow implemented in this study. At the time of initial treatment planning image (Day $1 \mathrm{CT}$ ) acquisition, the protrusion of the needles relative to the template surface is measured and recorded as the needle retraction depth. Prior to all delivered fractions, the needle retraction depth is verified to ensure needle insertion depths have not changed between fractions. This needle insertion depth measurement is illustrated in Figure 1B. If the needle retraction depth has changed, manual adjustments are made to equal the measurement that was recorded at the time of treatment planning image acquisition.

On Day 2, prior to the second fraction treatment, a CT (Day 2 CT) is acquired to facilitate assessment of

Table 1. Characteristics of patient and brachytherapy treatment

Characteristics

\begin{tabular}{lc}
\hline Age (years), median (range) & 68 (28-73) \\
\hline HR-CTV volume ( $\left.\mathrm{cm}^{3}\right)$, median (range) & 39.8 (24.8-92.0) \\
\hline Catheters ( $n$ of catheters), median (range) & 16 (14-18) \\
\hline Disease ( $n$ of patients) & 3 \\
\hline Primary cervix & 5 \\
\hline Primary vagina & 2
\end{tabular}

HR-CTV - high-risk clinical target volume 


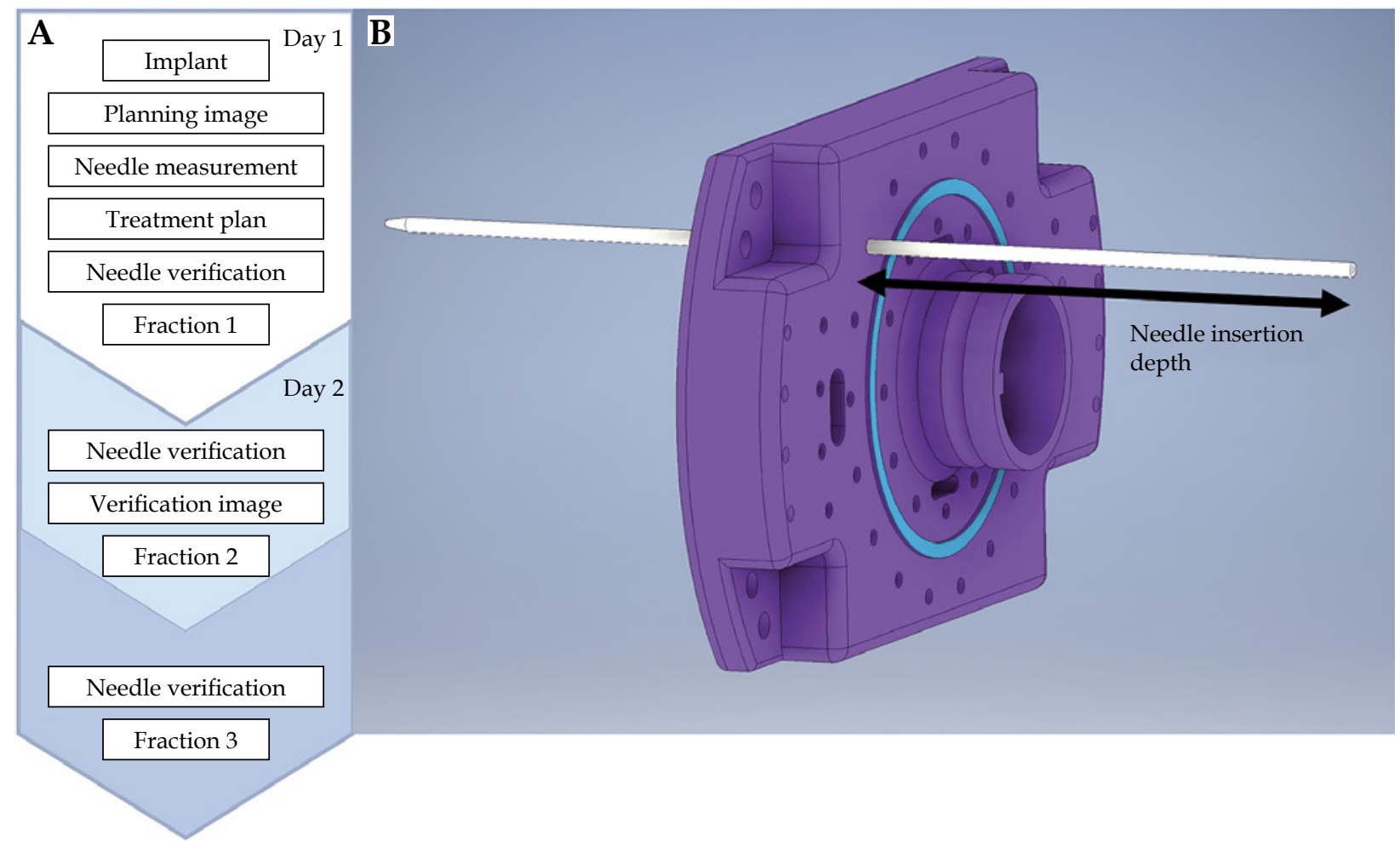

Fig. 1. Clinical verification workflow. A) The needle insertion depth measurement that is recorded at time of initial treatment planning image; B) Three-fraction, two-day, clinical workflow. The needle insertion depth verification is performed prior to delivery of fraction 2 and 3

inter-fraction motion that occurred overnight. The CT acquired before the delivery of the second fraction is registered to the planning CT that was acquired on Day 1. This process is conducted in the Varian Eclipse ${ }^{\mathrm{TM}}$ (Varian Medical Systems, Palo Alto, CA, USA) workspace using rigid registration.

The registration was performed with the use of automatic registration tool. The volume of interest was defined between the head of the femurs (left-right) and along the vaginal obturator to $1 \mathrm{~cm}$ above the most superior aspect of the CTV. The registration was assessed for agreement between rigid applicator features and identifiable anatomical features. Minor manual adjustments were made to improve agreement when necessary. The registered images are used to assess the Day 1 and Day 2 anatomical and implant congruence. Additional needle modifications are performed at this point in the workflow to best match the implant congruence between Day 1 and Day 2 images.

\section{Clinical treatment planning strategy}

With applicator in place, 1.5 Tesla T2 magnetic resonance imaging (Siemens, Berlin \& Munich, Germany) aided in contouring CTV, bladder, rectum, and sigmoid, while the Day 1 CT was used for implant reconstruction. Clinical treatment plans were produced in Oncentra ${ }^{\circledR}$ Brachytherapy (Elekta, Stockholm, Sweden) via inverse optimization followed by minor manual modifications to obtain optimal dosimetry. Brachytherapy treatment plan dose metrics were evaluated by the requirement for CTV D90\% to be greater than $100 \%$ of the prescription [1]. Normal tissue constraints are outlined in the EMBRACE II protocol, with a brief summary of planning aims and limits for prescribed dose highlighted in Table 2 [11,12]. These normal tissue constraints are similar to the recommendations of the ABS (also listed in Table 2) [1]. The cumulative patient dosimetry (termed the 'clinically intended plan') was determined by applying the first fraction treatment plan to subsequent fractions.

Table 2. Normal tissue constraints. The EMBRACE II planning aims (soft constraints) and limits for prescribed dose (hard constraints) and the American Brachytherapy Society guidelines

\begin{tabular}{lccc} 
Normal tissue & $\begin{array}{c}\text { EMBRACE II } \\
\text { Planning aims [11] }\end{array}$ & $\begin{array}{c}\text { EMBRACE II } \\
\text { Limits for prescribed dose [11] }\end{array}$ & $\begin{array}{c}\text { American Brachytherapy Society } \\
\text { Guidelines [1] }\end{array}$ \\
\hline Bladder $\mathrm{D}_{2 c c}$ & $<80 \mathrm{~Gy}_{3}$ & $<90 \mathrm{~Gy}$ & $<90 \mathrm{~Gy}_{3}$ \\
\hline Rectum $\mathrm{D}_{2 c c}$ & $<65 \mathrm{~Gy}_{3}$ & $<75 \mathrm{~Gy}_{3}$ & $<70 \mathrm{~Gy}_{3}$ \\
\hline Sigmoid $\mathrm{D}_{2 c c}$ & $<70 \mathrm{~Gy}$ & $<75 \mathrm{~Gy}$ & $<70 \mathrm{~Gy}_{3}$
\end{tabular}




\section{Retrospective comparative treatment planning study}

For each patient, the Day 2 CT verification image provided a visualization of the current position of the implant. On the Day 2 CT, target and normal tissue contours were produced as well as a newly digitized implant, which was used as the basis for the creation of Day 2 treatment plans. For the comparative treatment planning study, the cumulative dosimetry was determined by applying the weighted average of the Day 1 treatment plan (fraction 1) and Day 2 treatment plan (fraction 2 and 3). To evaluate inter-fraction changes, deviation from the clinically intended plan was reported. Two different strategies were performed to evaluate the intended dosimetry: a) clinically delivered dosimetry, and b) inter-fraction adapted dosimetry.

\section{Clinically delivered plan}

The first evaluation strategy transcribed the dwell positions and times from the Day $1 \mathrm{CT}$ to the Day $2 \mathrm{CT}^{\prime} \mathrm{s}$ reconstructed catheter geometry to reproduce the clinically intended dosimetry on the patient's Day 2 anatomy. This transcribed plan (termed 'clinically delivered plan') provided the best available estimate of the actual dose delivered to the patient based on the available CT datasets.

\section{Inter-fraction adapted plan}

The second evaluation strategy adapted the clinically delivered plan to optimize the dosimetry to the Day 2 anatomy and implant position. For a fair comparison to the Day 1 CT plan quality, the Day 2 plan was modified to match the original CTV coverage within $\pm 1 \mathrm{~Gy}_{10}$. With matched CTV coverage, the dose to normal tissues was minimized by manual adjustment of dwell times and positions. This optimized plan (termed the 'adapted plan') provided the best estimate of the improvement to normal tissue doses that could be made if inter-fraction adaptive planning was utilized.

\section{Evaluation criteria}

Two pair-wise analyses were performed (Prism GraphPad, San Diego, CA, USA) for the 10-patient cohort by comparing the clinically intended plan to both the clinically delivered and clinically adapted plans. The dose differences were described by the median and range, displayed as boxplots. Outliers in the analysis were specified when the dose difference was greater than $10 \mathrm{~Gy}_{3}$ for rectum and bladder, and $5 \mathrm{~Gy}_{3}$ for sigmoid. These dose difference thresholds were chosen based on the EMBRACE II minor variation criteria as a potentially meaningful difference in clinical outcome.

\section{Results}

\section{Clinically delivered plan}

The difference between the clinically intended plan and the clinically delivered plan is shown in Figure 2. For all patients, the median (range) difference from the intended CTV coverage was $1.4 \mathrm{~Gy}_{10}\left(0.3-4.4 \mathrm{~Gy}_{10}\right)$.
For all three normal tissues, the median (range) difference from the intended normal tissue dose was $2.6 \mathrm{~Gy}_{3}$ (0.1-15.5 $\left.\mathrm{Gy}_{3}\right)$. For two of 10 patients, the difference in a single normal tissue constraint exceeded $10 \mathrm{~Gy}_{3}$ from the intended dose (plotted in Figure 2 with symbols). Although this increase to normal tissue is substantial, the change did not lead to a violation of the ABS guidelines or the EMBRACE II limits for prescribed dose for any patients in the study.

\section{Adapted plan}

Figure 3 shows the difference between the adapted and clinically delivered plan for only normal tissues, as the target coverage was forced to match in the adaptive planning strategy. In two patients, normal tissue differences between intended and delivered that exceeded $10 \mathrm{~Gy}_{3}$ are displayed in Figure 2. These two patients also demonstrated (highlighted in Figure 3 with circle and triangle) the benefit that adaptive planning can have on improving the dose delivered to the CTV. In one patient (circle), the clinically intended plan quality was fully recovered to match the clinically intended dose to the bladder. In the other patient (triangle), the adaptive plan improved the distribution compared to the clinically delivered plan, but still resulted in an increase to the $\mathrm{D}_{2 \mathrm{cc}}$ of approximately $6 \mathrm{~Gy}_{3}$, indicating the anatomy on Day 2 was less favorable.

\section{Discussion}

The assessment of inter-fraction changes for ISBT treatments is important to provide clinicians confidence in the robustness of treatment. This template-based ISBT technique does not inherently include an inter-fraction

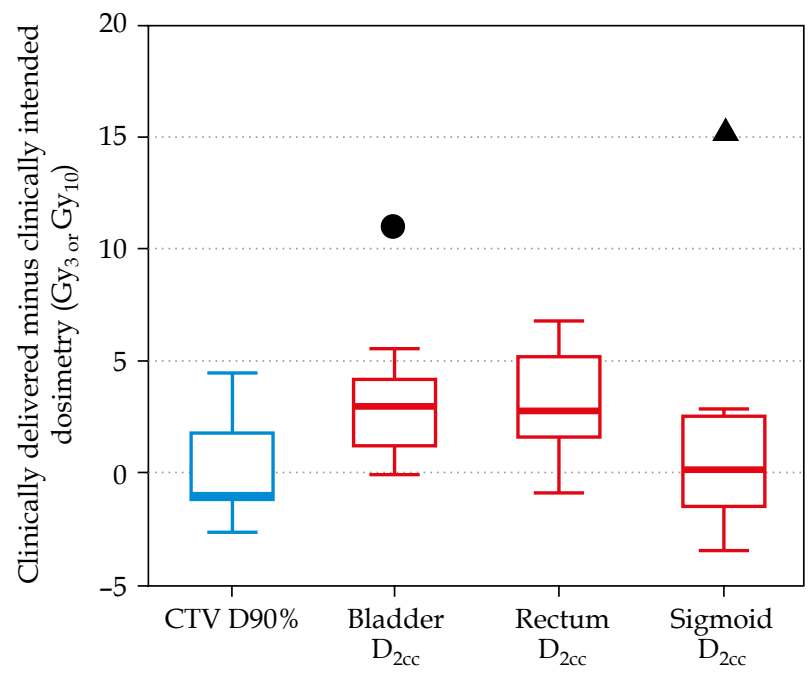

Fig. 2. Difference in 2-Gy-per-fraction equivalent dose $\left(\mathrm{EQD}_{2}\right)$ doses between the clinical delivered plan and the clinically intended plan for target volumes (blue) and normal tissues (red). The box encloses the median and displays the $25^{\text {th }}$ and $75^{\text {th }}$ percentile, while the error bars display the maximum and minimum changes (excluding the two patient outliers). The circle and triangle indicate two distinct patients where the normal tissue dose exceeded $10 \mathrm{~Gy}_{3}$ 


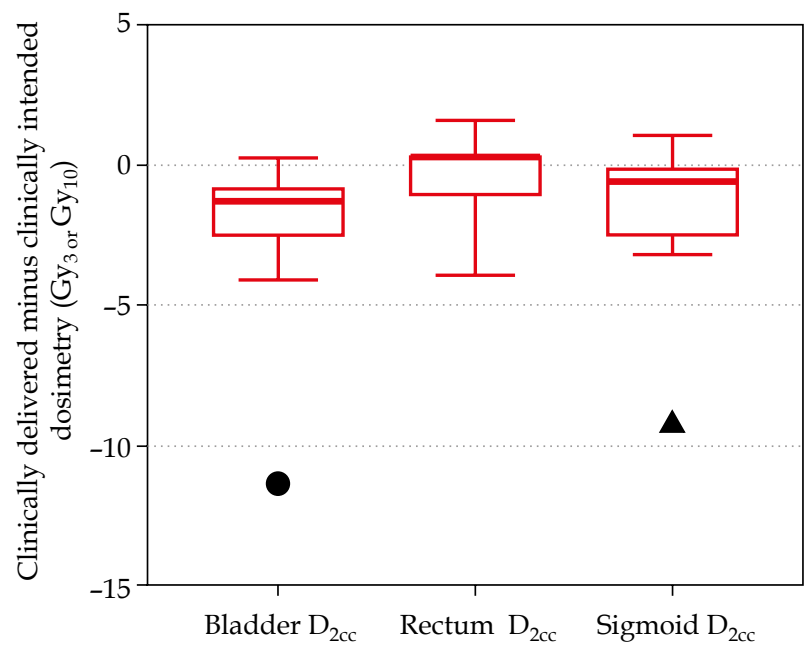

Fig. 3. Difference in 2-Gy-per-fraction equivalent dose $\left(\mathrm{EQD}_{2}\right)$ doses between the adapted plan and the clinically delivered plan for normal tissues (red). The box encloses the median and displays the $25^{\text {th }}$ and $75^{\text {th }}$ percentile, while the error bars display the maximum and minimum changes (excluding the patient outliers). The circle and triangle indicate two distinct patients corresponding to the same patients in Figure 2, where the normal tissue dose exceeded $10 \mathrm{~Gy}_{3}$ when comparing delivered and intended plans

adaptive planning component, as is the case with intracavitary ISBT that is usually performed with multiple, distinct implants and treatment plans. In this implementation, a single implant with the prescription of dose delivered over multiple implants on consecutive days resulted in clinically acceptable dose delivery. Validation of this treatment approach provides justification that implementation of perineal template-based ISBT with an ABS-guided prescription regimen will meet the normal tissue constraints specified by the ABS and the
EMBRACE II protocol without the need for inter-fraction adaptive planning.

This implementation of template-based ISBT treatments does not include imaging prior to the third fraction. The study published by Gladwish et al. [10] treated the second fraction without imaging verification; however, they utilized a second image to determine if re-planning is warranted on the third fraction. The institutional workflow presented in this study acquires a CT image prior to the second fraction and therefore, allows flexibility with the clinical decision to treat the second fraction and re-plan the third or, alternatively, re-plan prior to treating the second fraction. These results suggest that for any three-fraction prescription regimen, a Day 2 verification image adds value to assessing inter-fraction changes. The results of this study also reinforce the findings of previous adaptive planning studies indicating that re-planning may provide value in particular cases $[7,8,9,10]$. That is, adaptive planning is capable of improving plan quality, but is not strictly required to adhere to the ABS normal tissue guidelines or the EMBRACE II limits for prescribed dose.

For the CTV D90\%, changes in dosimetry due to inter-fraction motion were observed to closely match their clinically intended dosimetry. Only three of 10 patients exceeded a change of $\pm 2 \mathrm{~Gy}_{10}$, with a maximum change of $4.4 \mathrm{~Gy}_{10}$. This result is encouraging and provides confidence to clinicians that large dosimetric deviation from intended dosimetry is limited. Similarly, observed differences in normal tissue dosimetry were generally minimal. In cases with large inter-fraction normal tissue dosimetric changes between the delivered and intended dosimetry, the anatomy was initially favorable. Although the degradation observed in these cases is undesirable, it did not lead to a violation of the normal tissue constraints.

For the two patients with changes in delivered normal tissue dosimetry in excess of $10 \mathrm{~Gy}_{3}$, inconsistencies in rectal and sigmoid gas filling were responsible. An

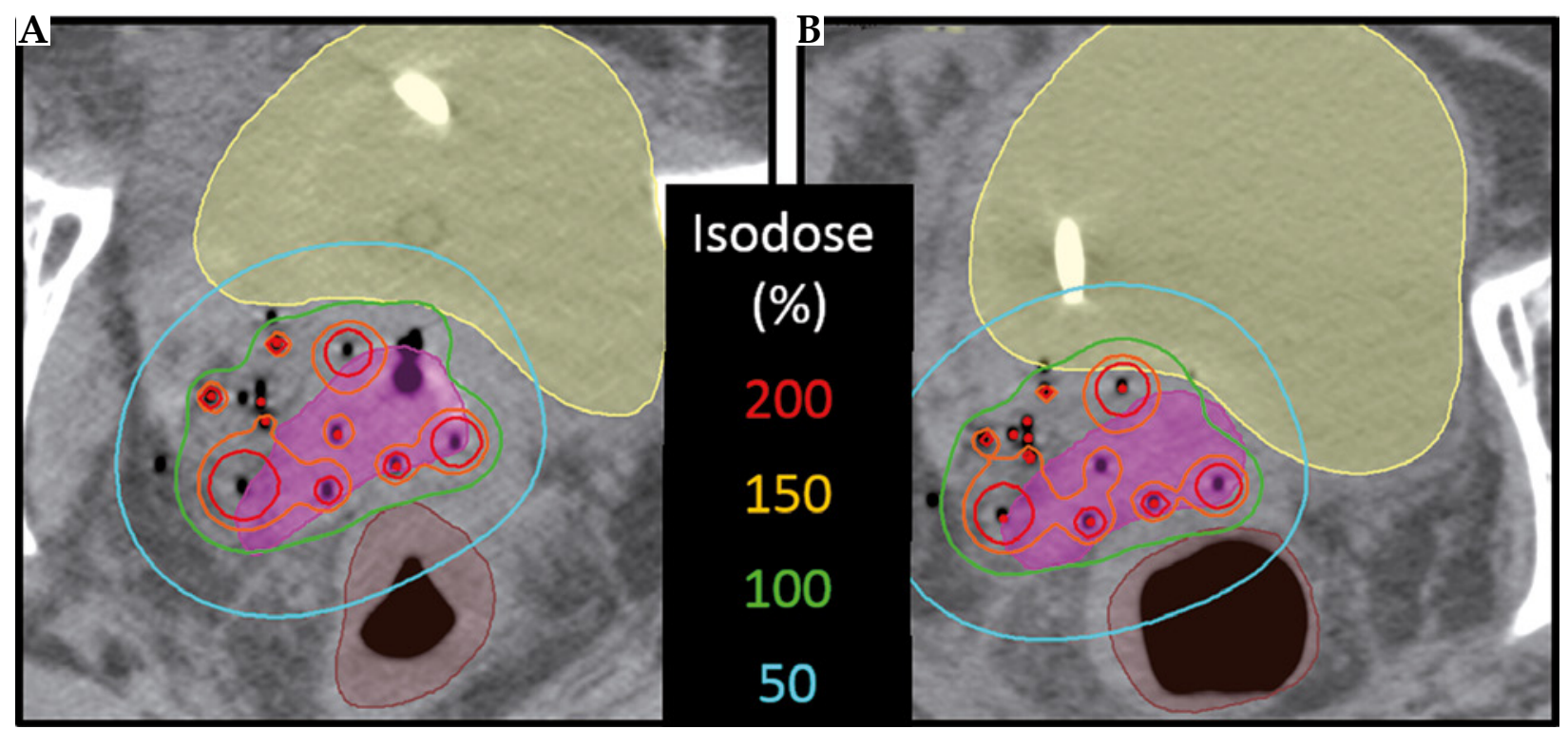

Fig. 4. Comparison of inter-fraction anatomical changes, with increased gas in the rectum on Day 2 resulting in higher dose to normal tissues. A) Day 1 CT and clinically intended plan, and (B) Day 2 CT and clinically delivered plan. The CTV (pink), bladder (yellow), and rectum (brown) are displayed 
example is shown in a representative patient (Figure 4), in which changes to rectal gas are evident. Even though draining rectal gas is routinely applied in practice for ISBT treatments, it is difficult to predict the dosimetric impact of anatomical changes without assessment by verification imaging. For these two patients, neither received a total dose to either bladder or sigmoid that exceeded the ABS guidelines or the EMBRACE II limits for prescribed dose. However, this result should motivate clinicians to not only meet normal tissue dose constraints during initial planning but rather aim to improve upon them when possible, as inter-fraction anatomical changes may increase delivered dose compared to the intended dose. Consequently, in cases where Day 1 dosimetry is approaching the limit of normal tissue planning aims, care should be taken to assess Day 2 changes and - resources permitting - may prompt an adaptive plan.

\section{Conclusions}

For perineal template-based gynecologic ISBT, the implementation of an ABS-guided three-fraction prescription regimen with a single treatment plan can be delivered to meet the ABS guidelines and the EMBRACE II protocol limits for prescribed dose. This ISBT implementation provides steps to promote implant reproducibility as well as recommends a Day 2 verification image that aims to assist clinicians in identifying large inter-fraction anatomical changes.

\section{Disclosure}

Authors report no conflict of interest.

\section{References}

1. BeriwalS, Demanes DJ, Erickson B et al. American Brachytherapy Society consensus guidelines for interstitial brachytherapy for vaginal cancer. Brachytherapy 2012; 11: 68-75.

2. Mock U, Kucera H, Fellner C et al. High-dose-rate (HDR) brachytherapy with or without external beam radiotherapy in the treatment of primary vaginal carcinoma: Long-term results and side effects. Int J Radiat Oncol Biol Phys 2003; 56: 950-957.

3. Rodriguez S, Otal A, Richart J et al. Pre-plan technique feasibility in multi-interstitial/endocavitary perineal gynecological brachytherapy. J Contemp Brachytherapy 2017; 5: 472-476.

4. Brown DW, Damato AL, Sutlief S et al. A consensus-based, process commissioning template for high-dose-rate gynecologic treatments. Brachytherapy 2016; 15: 570-577.

5. Karlsson L, Thunberg P, With A et al. 3D image-based adapted high-dose-rate brachytherapy in cervical cancer with and without interstitial needles: measurement of applicator shift between imaging and dose delivery. J Contemp Brachytherapy 2017; 1: 52-58

6. Dumane VA, Yuan Y, Sheu RD et al. Computed tomography-based treatment planning for high-dose-rate brachytherapy using the tandem and ring applicator: influence of applicator choice on organ dose and inter-fraction adaptive planning. J Contemp Brachytherapy 2017; 3: 279-286.

7. Lee S, Rodney E, Traughber B et al. Evaluation of interfractional variation of organs and displacement of catheters during high-dose-rate interstitial brachytherapy for gynecologic malignancies. Brachytherapy 2017; 16: 1192-1198.
8. Damato AL, Cormack RA, Viswanathan AN. Characterization of implant displacement and deformation in gynecologic interstitial brachytherapy. Brachytherapy 2014; 13: 100-109.

9. Rey F, Chang C, Mesina C et al. Dosimetric impact of interfraction catheter movement and organ motion on MRI/ CT guided HDR interstitial brachytherapy for gynecologic cancer. Radiother Oncol 2013; 107: 112-116.

10. Gladwish A, Ravi A, Barbera L et al. Characterizing the impact of adaptive planning on image-guided perineal interstitial brachytherapy for gynecologic malignancies. Brachytherapy 2018; 17: 352-359.

11. Pötter R, Tanderup K, Kirisits $C$ et al. The EMBRACE II study: The outcome and prospect of two decades of evolution within the GEC-ESTRO GYN working group and the EMBRACE studies. Clin Transl Radiat Oncol 2018; 9: 48-60.

12. Sturdza A, Pötter R, Fokdal LU et al. Image guided brachytherapy in locally advanced cervical cancer: Improved pelvic control and survival in RetroEMBRACE, a multicenter cohort study. Radiother Oncol 2016; 120: 428-433.

13. Viswanathan AN, Beriwal S, De Los Santos JF et al. American Brachytherapy Society consensus guidelines for locally advanced carcinoma of the cervix. Part II: High-dose-rate brachytherapy. Brachytherapy 2012; 11: 47-52. 